\title{
EchoGéo
}

\section{La géoarchéologie fluviale}

Concepts, attendus et méthodes d'étude rétrospectives appliqués à la caractérisation du risque hydrologique en domaine méditerranéen

\section{Gilles Arnaud-Fassetta}

\section{OpenEdition}

\section{Journals}

Édition électronique

URL : https://journals.openedition.org/echogeo/2187

DOI : $10.4000 /$ echogeo. 2187

ISSN : 1963-1197

Éditeur

Pôle de recherche pour l'organisation et la diffusion de l'information géographique (CNRS UMR 8586)

Référence électronique

Gilles Arnaud-Fassetta, « La géoarchéologie fluviale », EchoGéo [En ligne], 4 | 2008, mis en ligne le 05 mars 2008, consulté le 31 juillet 2021. URL : http://journals.openedition.org/echogeo/2187 ; DOI :

https://doi.org/10.4000/echogeo.2187

Ce document a été généré automatiquement le 31 juillet 2021.

EchoGéo est mis à disposition selon les termes de la licence Creative Commons Attribution - Pas d'Utilisation Commerciale - Pas de Modification 4.0 International (CC BY-NC-ND) 


\title{
La géoarchéologie fluviale
}

\author{
Concepts, attendus et méthodes d'étude rétrospectives appliqués à la \\ caractérisation du risque hydrologique en domaine méditerranéen
}

\author{
Gilles Arnaud-Fassetta
}

La géoarchéologie fluviale, un apport substantiel dans l'appréciation des liens sociétésmilieux

1 La " géoarchéologie ", terme qu'il faut préférer à celui de " géologie archéologique " (Rapp et Gifford, 1982), se place à l'interface entre les sciences de la Terre (qui incluent la géographie physique) et les sciences s'intéressant aux activités humaines passées (Davidson et Shackley, 1976). La géoarchéologie a eu une grande influence en Amérique du Nord, en Europe du Nord-ouest, en Afrique du Nord-est, en Asie Mineure et au Moyen-Orient, en raison de la présence de nombreux sites archéologiques dont les vestiges ont été découverts interstratifiés dans les vieux dépôts de plaines alluviales ou deltaïques.

2 L'approche géoarchéologique se place dans un cadre temporel assez long, en général celui de l'Holocène (Roberts, 1998), avec des résolutions parfois très fines, de l'ordre de la décennie selon les périodes considérées et la qualité des marqueurs chronostratigraphiques. Sa finalité n'est pas seulement de définir les conditions environnementales des sociétés du passé (i.e., valeurs patrimoniale et culturelle), mais d'apporter aussi une réflexion sur les conditions environnementales du passé qui puisse servir à mieux comprendre le présent et, pourquoi pas, à mieux anticiper le futur (cf. par exemple le débat sur la modification possible du réseau hydrographique dans le delta du Rhône suite aux graves inondations de 2003, à la lumière de ce que l'on sait, par les travaux de recherche géoarchéologiques, du fonctionnement et du tracé des paléochenaux antiques et médiévaux).

3 La géoarchéologie implique forcément d'avoir une bonne connaissance des clés permettant de décrire les paléoenvironnements, d'expliquer les causes (climatiques, anthopiques, autocyclicité) de leur évolution et de juger de la vulnérabilité sociétale face aux contraintes hydroclimatiques. Ce type de recherches pluri- ou transdisciplinaires s'insère dans le vaste champ des enquêtes paléoenvironnementales qui ont connu ces dernières années une grande expansion internationale dans la définition 
des relations homme-milieu-climat, aussi bien en France (Bravard et Presteau, 1997 ; Arnaud-Fassetta et Landuré, 2003 ; Burnouf et Leveau, 2004) que dans le reste du bassin méditerranéen (Raban et Holum, 1996 ; Vermeulen et De Dapper, 2000 ; ArnaudFassetta et al., 2003 ; Fouache, 2003 ; Morhange et Provansal, 2007) pour ne citer que les publications traitant des systèmes fluviaux et des ports.

Très en vogue ces dernières années, la géoarchéologie fluviale n'aurait pas connu un tel essor si les sociétés humaines n'avaient pas très tôt été attirées par les plaines alluviales. Les milieux rivulaires sont généralement riches en ressources alimentaires (poissons, oiseaux, mammifères) ; ils sont d'autant plus variés que le paysage fluvial est complexe. Bravard et Salvador (1999) ont également souligné le rôle des fonds de vallée, comme terres agricoles (fertilité et humidité), axes de circulation privilégiés (transport de marchandises et du bois), lieux de production d'énergie dérivée des aménagements hydrauliques (dès le Moyen Age) et espaces refuges à des groupes humains (Danube/Hongrie au XVIIIe siècle ; zones humides en régions arides : Mésopotamie, bassins du Nil et de l'Indus).

Les plaines alluviales sont donc considérées comme des milieux " attractifs » et ce, malgré le risque hydrologique, qui se décompose en trois sous-catégories : le risque phréatique (nappes hautes/drainage, salinisation des sols), en liaison avec la mise en valeur agricole, le risque d'érosion et le risque d'inondation. En fait, elles correspondent à des milieux faits de contraintes «naturelles» auxquelles l'Homme s'est souvent adapté.

6 L'un des objectifs des recherches géoarchéologiques est justement de déterminer l'importance des contraintes " naturelles " à chaque étape de l'occupation des rives d'un cours d'eau. Ces contraintes s'expriment à travers un bilan en eau et en sédiments soit excédentaire (crue, hydromorphie, défluviation), soit déficitaire (assèchement des plaines d'inondation). La difficulté tient au fait que les actions anthropiques concourent plus ou moins directement à ce bilan hydrosédimentaire, ce qui perturbe, à l'échelle de l'Holocène, la détermination des paramètres naturels (climat, géologie) qui sous-tendent l'évolution des milieux fluviaux. Dès lors, tenter de faire la part de l'anthropisation et du climat dans l'évolution des milieux fluviaux relève d'une tâche difficile (Arnaud-Fassetta, 2007).

7 L'état actuel des recherches géoarchéologiques permet également de s'interroger sur les relations que les sociétés (rurales ou urbaines) du passé, venues s'installer à proximité des grands cours d'eau, ont entretenu avec le risque fluvial (Arnaud-Fassetta, 2000 ; Fouache, 2003 ; Bravard, 2004 ; Carcaud, 2004 ; Allinne, 2005 ; Salvador, 2005). A l'échelle des temps historiques, ces recherches intègrent les concepts de seuil et de crise. Le concept de seuil, qui permet par exemple d'expliquer les ruptures dans la façon de s'exposer et de gérer le risque, reste étroitement lié à l'évolution des contraintes fluviales, elles-mêmes dépendantes de la variabilité hydroclimatique et de la morphogenèse en général. Pour les paléoenvironnementalistes, une crise hydrologique (ou un régime dominé par les crues durant plusieurs décennies à quelques siècles) s'explique avant tout par une succession d'aléas hydroclimatiques plus fréquents et de magnitude plus élevée qu'auparavant. Cependant, l'impact des crues peut être absorbé par la société si sa résilience est forte. Inversement, les effets d'une crise hydrologique peuvent être exacerbés dans un contexte de faible résilience sociétale, qui dépend du degré de vulnérabilité. Ainsi, le risque fluvial peut être perçu 
et vécu différemment au cours des siècles sans que ne se produisent forcément des ruptures hydrodynamiques.

Parvenir à résoudre ces questions nécessite le développement d'une approche pluridisciplinaire et un traitement de données multi-critères produites par les historiens, les archéologues et les naturalistes. Ainsi, l'aléa hydrologique est généralement étudié à partir de diverses sources historiques et de données sédimentaires acquises lors des fouilles géoarchéologiques. La quantification de la variabilité de l'aléa permet de réfléchir en termes de fréquence et de magnitude des crues et de leurs variations possibles dans le temps. Les variations climatiques peuvent modifier le régime d'un fleuve et créer, à proximité de celui-ci, des conditions plus ou moins contraignantes pour les sociétés riveraines. L'abandon d'un habitat peut être le signe (ou pas) d'un accroissement des contraintes hydrologiques, tant par excès d'eau douce (crues, hydromorphie) que par défaut (déficit hydrique, salinisation des terres). La géoarchéologie permet de retracer la nature et l'état de ces contraintes tout autant que la façon dont elles ont pu être gérées par les sociétés.

L'ajustement nécessaire des méthodes hydrogéomorphologiques aux contextes géoarchéologiques

9 Au cours des dernières décennies, la géomorphologie fluviale a eu de nombreuses applications dans le vaste champ des interactions entre les sociétés et leur environnement (Vita-Finzi, 1969 ; Butzer, 1982 ; Needham et Macklin, 1992 ; Bravard et Presteau, 1997 ; Brown, 1997 ; Vermeulen et De Dapper, 2000 ; Fouache, 2001). De nombreux points communs émergent de ces études, en particulier le recours aux trois approches hydromorphométriques classiquement utilisées en géomorphologie fluviale (hydrographie, hydrologie, hydraulique). Cependant, l'analyse critique de la littérature montre que ces trois approches n'ont pour ainsi dire jamais été conduites simultanément en géoarchéologie fluviale. La méthodologie développée ici propose justement de mener de front les reconstitutions paléohydrographiques, paléohydrologiques et paléohydrauliques. Les données sources sont les archives textuelles et iconographiques, les images récentes (photographies aériennes, images satellites, cartes) et les relevés de terrain, appliqués à deux deltas méditerranéens (Rhône, Isonzo ; figure 1). 
Figure 1. Localisation des sites étudiés

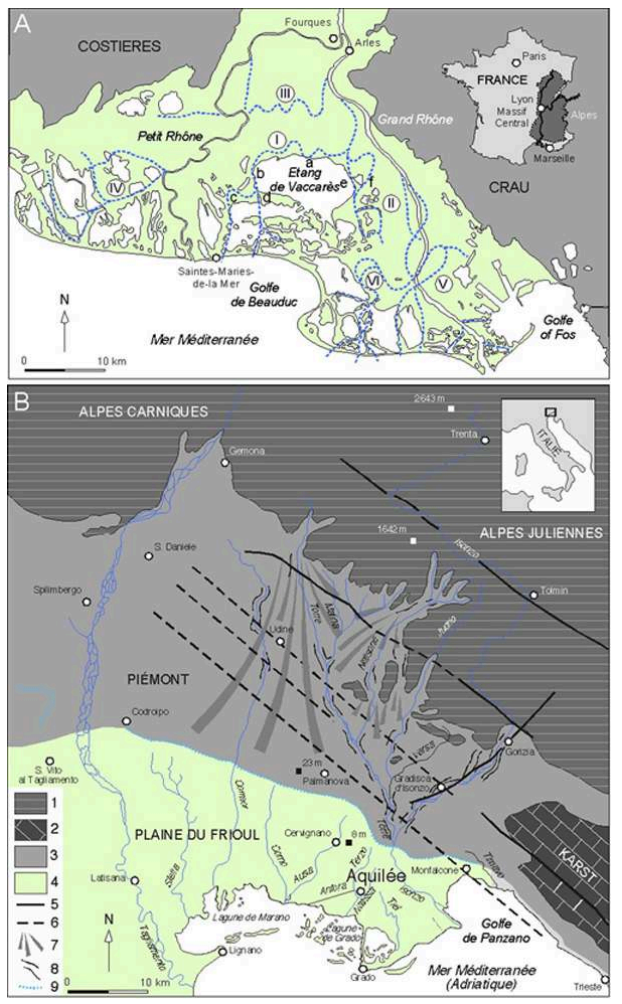

A : Le delta du Rhône. I = Rhône de Saint-Ferréol ; II = Rhône d'Ulmet ; III = Rhône d'Albaron ; IV = Rhône de Peccaïs ; $V$ = Rhône du Grand Passon ; VI = Rhône du Bras de Fer ; $a$ = site de Cabassole ; $b=$ site du Carrelet $; c=$ site des Combettes $; d$ = site de Mornès ; $=$ = site du Pont Noir $; f=$ site de La Capelière. B : La plaine deltaïque d'Aquilée et sa région [d'après Arnaud-Fassetta et al., 2003]. 1 = massifs montagneux calcaires et dolomitiques ; 2 = plateaux et massifs calcaires du Karst ; $3=$ piémont (marnes calcaires, calcaires marneux, flyschs marno-calcaires) ; 4 = plaine (remblaiement fluvio-marin quaternaire) $; 5=$ faille principale ; 6 = faille supposée (tracé hypothétique) $; 7=$ cône alluvial ; 8 = vallée encaissée (non représentée dans les unités montagneuses) ; 9 = ligne de résurgence karstique

La paléohydrographie, comme base de définition de l'image des cours d'eau

L'objectif de l'analyse paléohydrographique est de cartographier les anciens réseaux de drainage. Les cartes paléohydrographiques du delta du Rhône (Arnaud-Fassetta, 2000) et de la plaine d'Aquilée (Arnaud-Fassetta et al., 2003) ont été déduites de l'interprétation des données géomorphologiques, stratigraphiques et géoarchéologiques.

11 Les données géomorphologiques sont issues de l'analyse détaillée des cartes topographiques/géologiques (échelle $1 / 25.000$ à $1 / 50.000$ ) couplée à la photointerprétation. Selon la méthode exposée in Siché et al. (2006), les paléochenaux fluviatiles de la plaine d'Aquilée ont été extraits d'un modèle numérique de terrain par photogrammétrie digitale et intégrés à un SIG. Dans chacune des plaines deltaïques, la densité de drainage (en $\mathrm{km} / \mathrm{km}^{2}$ ) a été quantifiée en utilisant l'équation de Horton (1932).

12 Les données stratigraphiques ont été acquises pour l'essentiel à partir de carottes profondes (long. 5-25 m) prélevées le long de transects perpendiculaires à l'axe d'écoulement des paléochenaux (figure 2). Les milieux de sédimentation et les lithofaciès ont été définis en ayant recours aux techniques d'analyse classiques préconisées par Leopold et al. (1964) et Miall (1996). Les changements verticaux et 
latéraux de faciès sont montrés par les variations granulométriques, l'évolution des structures sédimentaires, les composants biogéniques et l'abondance du matériel organique.

Figure 2. Acquisition des données hydrogéomorphologiques

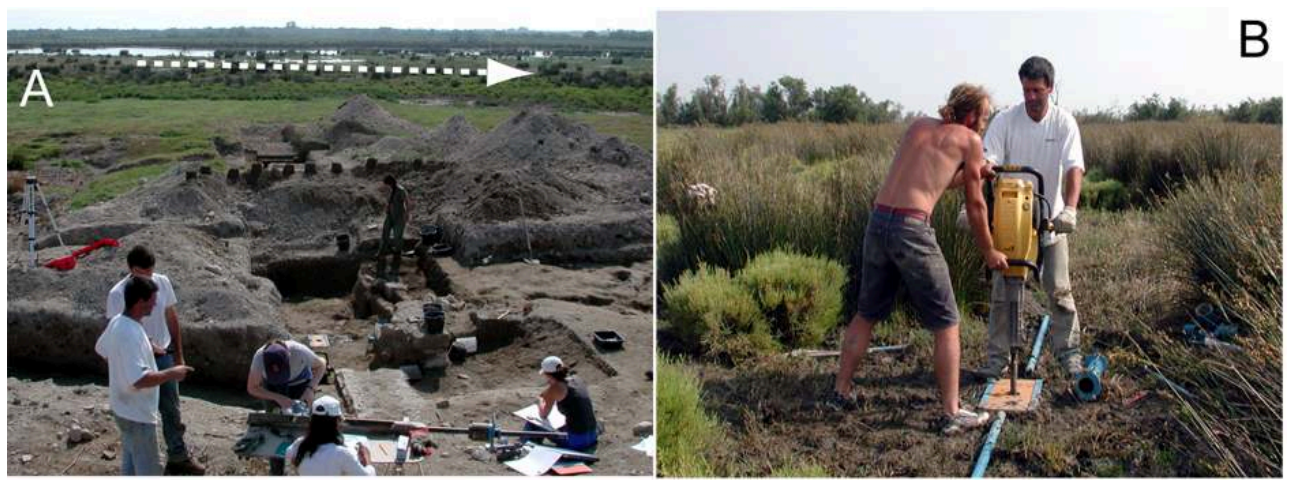

A : Fouilles géoarchéologiques et acquisition des données sédimentaires par le biais de sondages et de coupes stratigraphiques (site de l'Abbaye d'Ulmet, delta du Rhône ; cliché M. Charlet, 2002). B : L'utilisation d'une tarière motorisée appartenant à l'université Paris 7/PRODIG (site de l'Abbaye d'Ulmet, delta du Rhône ; cliché M. Charlet, 2002).

Les unités sédimentaires ont été datées par la méthode radiocarbone et les vestiges archéologiques. Ces derniers sont quelquefois de bons indicateurs paléohydrographiques (ponts, structures portuaires, épaves).

La paléohydrologie, comme outil de caractérisation de la dynamique fluviale La paléohydrologie a pour but de caractériser la dynamique fluviale et plus particulièrement le régime des cours d'eau. Une méthode multi-critères, basée sur le croisement de plusieurs types de données acquises au moyen de carottages, de sondages à la tarière et de coupes stratigraphiques, a permis de définir les critères d'identification du régime hydrosédimentaire des cours d'eau, à partir des dépôts du chenal et de sa plaine d'inondation adjacente (Arnaud-Fassetta, 2000).

Ce travail reprend en partie la typologie des régimes établie par Erskine et Warner (1988), pour lesquels le régime d'un cours d'eau subit des variations qui le font successivement passer d'un "régime dominé par les crues " (flood-dominated regime) à un " régime dominé par les basses eaux " (drought-dominated regime). En réalité, j'ai adapté cette typologie à la complexité du régime hydrologique méditerranéen. Finalement, les reconstitutions paléohydrologiques m'ont permis de définir trois types de régime : entre les deux précédents s'intercalent les « régimes dominés par des crues irrégulières » (Arnaud-Fassetta, 2000).

Les reconstitutions paléohydrologiques sont basées sur l'analyse stratigraphique des sédiments de la plaine alluviale et deltaïque (nature du chenal, migration des formes; Arnaud-Fassetta, 2006). En particulier, l'évolution de la « compétence maximale » a pu être suivie en extrayant des courbes granulométriques le grain médian $\left(D_{50}\right)$ des sédiments de chenal, que l'on a mis en fonction avec le percentile le plus grossier $\left(D_{99}\right)$, selon la méthode de Passega (1957). L'utilisation du critère de compétence a été complétée par l'estimation des paramètres hydrauliques.

La paléohydraulique, comme outil additionnel dans les hydrosystèmes méditerranéens

17 Dans les bassins méditerrannéens, les crues peuvent être très brutales (crues éclairs) et puissantes et la reconstitution de leurs caractéristiques hydrauliques permet de fournir 
une bonne information sur leur occurrence par le passé. La méthode utilisée pour reconstituer les conditions paléohydrauliques des cours d'eau a été présentée in Arnaud-Fassetta (2007) : j'en rappelle ci-dessous les principales étapes.

Les reconstitutions paléohydrauliques ont nécessité l'acquisition de plusieurs sections transversales dont la géométrie a été déduite des sections transversales reconstituées (carte topographique, sondages électriques, carottes sédimentaires. Avec les données stratigraphiques, elles ont permis de quantifier plusieurs paramètres hydrauliques (à

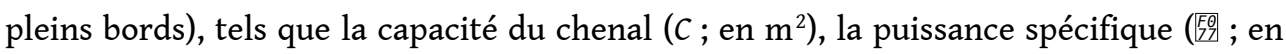
$\mathrm{W} / \mathrm{m}^{2}$ ), qui permet de quantifier la capacité de travail des cours d'eau, laquelle contrôle aussi leur instabilité latérale (Nanson et Croke, 1992), et le débit ( $Q$; en $\left.\mathrm{m}^{3} / \mathrm{s}\right)$ estimé à partir de la moyenne des résultats obtenus avec trois équations, celles de Manning (1891), de Williams (1978) et de Rotnicki (1991). L'estimation du coefficient de rugosité de Manning ( $n$; adimensionnel) a été nécessaire pour résoudre la dernière équation. Sa valeur a été déterminée par la formule de Strickler (1923) sur le Rhône et par la moyenne des résultats obtenus par l'équation de Strickler (1923), la table de Chow (1959) et la formule de Limerinos (1970) sur l'Isonzo.

19 En conclusion, les trois approches (paléohydrographie, paléohydrologie, paléohydraulique) reposent sur des méthodes qui leur sont propres et sur des attendus complémentaires. L'intérêt de les mener de front a permis d'obtenir des résultats intéressants sur la caractérisation de la dynamique fluviale du Rhône et de l'Isonzo, replacée dans le cadre physique et humain de leur delta respectif.

L'analyse rétrospective du risque fluvial en milieu rural et urbain à travers les exemples des deltas du Rhône (France du Sud) et de l'Isonzo (Italie du Nord)

Par rapport à cette réflexion complexe sur le risque fluvial sont ici présentées deux études de cas centrées sur des sites deltaïques nord-méditerranéens (figure 1) : l'un à dominante rurale (le delta du Rhône), l'autre urbaine (Aquilée). L'histoire de ces deux sites s'est forgée à travers des siècles de lutte contre des aléas hydrologiques dont l'impact a varié en fonction du contexte climato-anthropique. La vulnérabilité sociétale a pu également évoluer pour de multiples raisons (économique, technique, sociale, politique, hydroclimatique). Les deux secteurs choisis présentent à la fois l'intérêt d'enrichir la connaissance sur les fluctuations paléohydrologiques à l'échelle de l'Europe occidentale, d'approfondir les relations systémiques entre les fleuves et les sociétés urbaines et rurales et d'apporter des éléments nouveaux sur l'histoire des aménagements hydrauliques dans les deltas.

21 Ainsi, il ressort que la façon de s'affranchir des effets de l'aléa hydrologique et de gérer le risque fluvial a été distincte, selon que l'on soit en ville ou à la campagne. Les travaux menés dans le delta du Rhône ont montré qu'à la campagne, l'aménagement hydraulique de la plaine, durant l'Antiquité et le haut Moyen Age, s'est fait au travers de l'emploi de " techniques douces » (figure 3), ne modifiant qu'a minima le milieu (on compose avec le milieu " naturel »). L'intervention plus lourde sur l'hydrographie ne se fera que plus tard, à partir du bas Moyen Age. 
Figure 3. Quelques exemples d'équipements et de structures hydrauliques antiques découverts dans le delta du Rhône

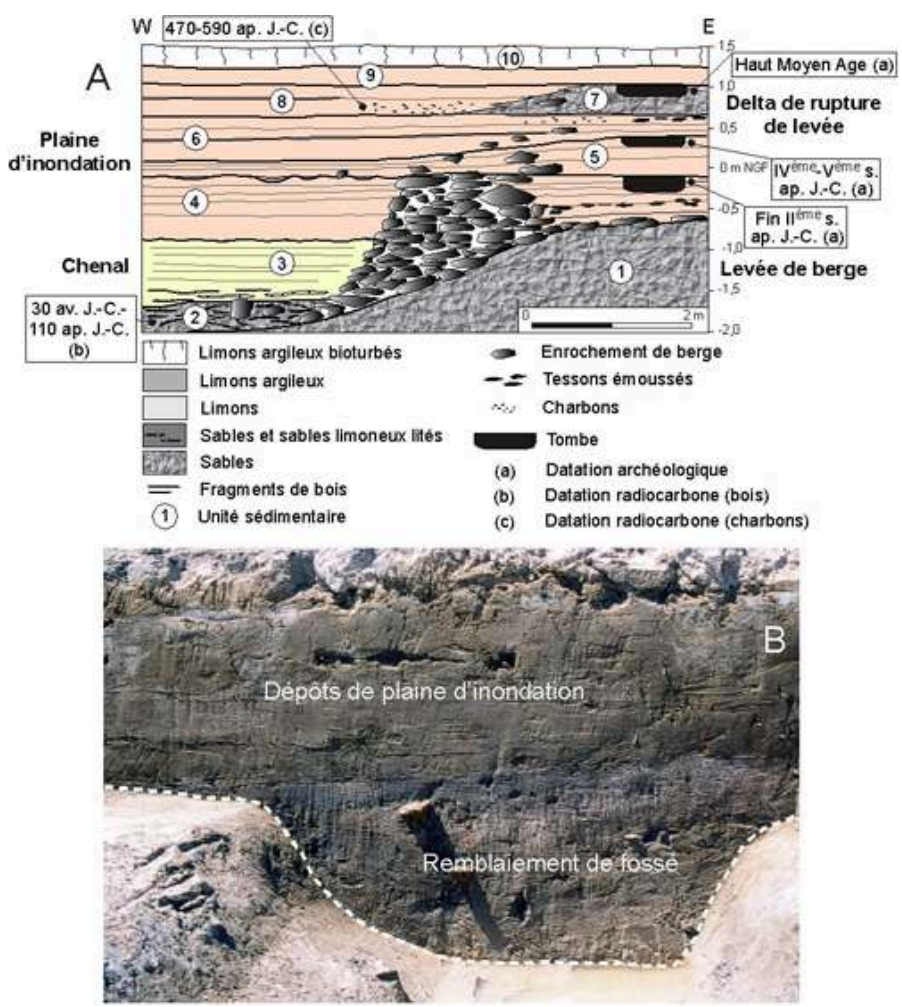

A : Coupe stratigraphique du Carrelet montrant la présence d'une levée de berge supportant un enrochement hydraulique daté de 30 av. J.-C.-110 apr. J.-C. [d'après Arnaud-Fassetta, 2000]. B : Fossé de drainage (Ier siècle av. J.-C.) découvert sur le site de La Capelière (cliché C. Landuré, 2001).

Les lieux d'installation de l'habitat sont choisis en fonction de la proximité des bourrelets alluviaux et des levées de berge, qui font gagner l'altitude nécessaire (quelques décimètres à quelques mètres) pour s'affranchir des inondations banales (figure 4). 
Figure 4. Paléohydrographie et occupation du sol en Camargue [d'après Arnaud-Fassetta et Landuré, 2003].

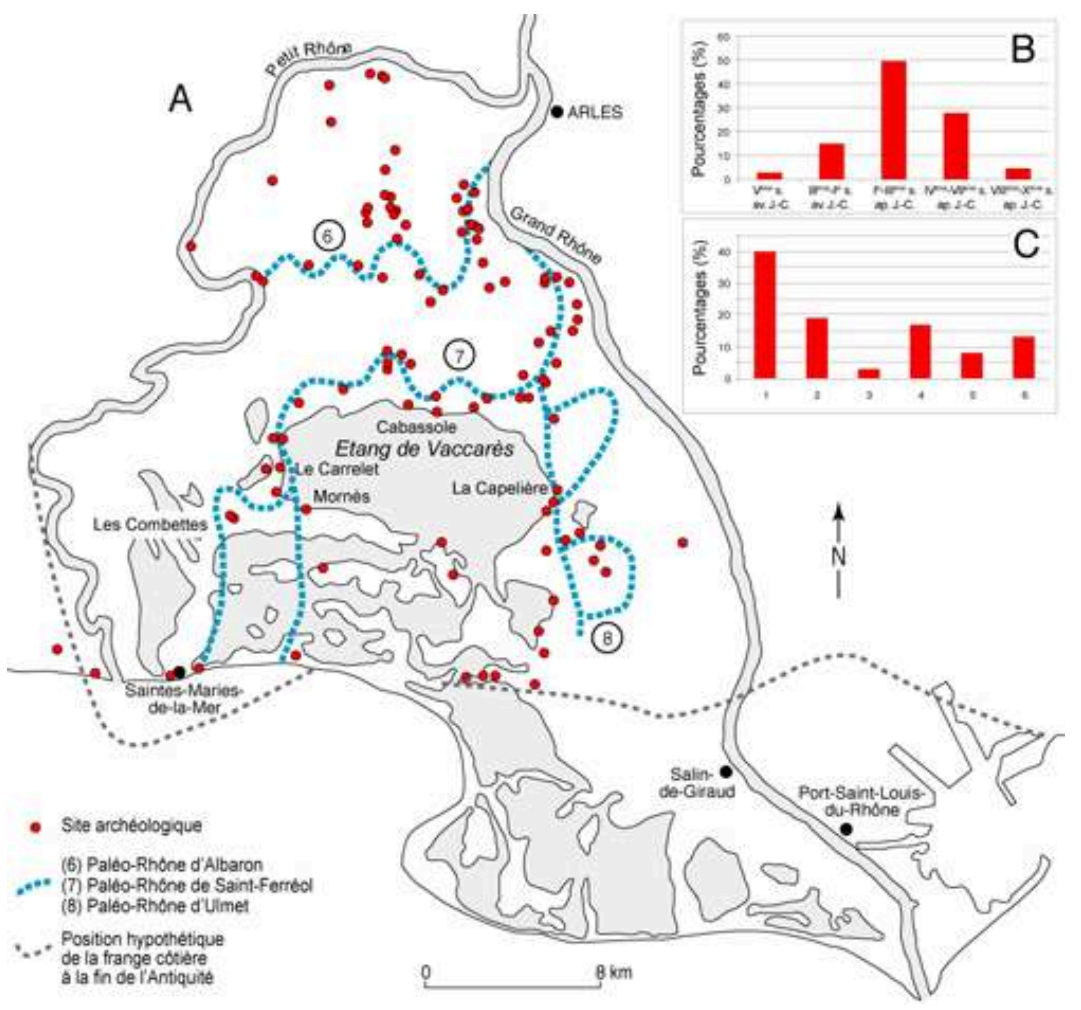

A : Carte générale des sites archéologiques recensés en Camargue entre le Ve siècle av. J.-C. et le $\mathrm{X}^{\mathrm{e}}$ siècle apr. J.-C. B : Evolution du nombre (exprimé en \%) de sites archéologiques entre le $\mathrm{V}^{\mathrm{e}}$ siècle av. J.-C. et le $\mathrm{X}^{\mathrm{e}}$ siècle apr. J.-C. C : Localisation des sites archéologiques dans la plaine deltaïque. 1 = sites à proximité du Rhône de Saint-Ferréol ; 2 = sites à proximité du Rhône d'Ulmet ; 3 = sites à proximité de la diffluence entre le Rhône de Saint-Ferréol et le Rhône d'Ulmet ; 4 = sites à proximité du Rhône d'Albaron ; 5 = sites à proximité du rivage côtier ; 6 = autres sites.

Le but étant aussi de contrôler et exploiter au mieux les ressources naturelles (eau, sel, bois, pêche, ...), on reste donc au plus près du fleuve. Les actions se limitent à l'amélioration des conditions de drainage dans la plaine d'inondation et à la consolidation des berges en bordure des chenaux s'écoulant à proximité des villae. La vulnérabilité reste donc forte lorsque l'aléa devient plus fréquent et plus morphogène. Pourtant, il n'existe pas de déterminisme strict et permanent du milieu, même si les sites habités ne sont pas à l'abri de catastrophe (inondation brutale par rupture de berge) lors de certaines phases de « régime dominé par les crues " (figure 5).

En ville, à l'image du site d'Aquilée, les mesures prises pour limiter l'impact des crues ont été souvent plus précoces (début de l'Antiquité), plus radicales et quelquefois irréversibles quant au fonctionnement hydrosédimentaire et aux impacts sur le milieu : chenalisation, contraction du lit fluvial, curage et dragage, remblaiement artificiel de chenaux (figure 6). En ville, les enjeux sont évidemment tout autres qu'à la campagne et les moyens mis en œuvre sont plus conséquents, afin d'assurer la pérennité du site, des aménagements et d'un grand nombre de personnes. Le risque fluvial est réel mais il est considéré comme secondaire par rapport aux bénéfices (économique, stratégique, politique) que l'on peut tirer de l'exploitation et de l'utilisation du Natisso. 
Figure 5. Résultats des récentes fouilles géoarchéologiques à La Capelière, delta du Rhône [d'après Arnaud-Fassetta et Landuré, 2003]

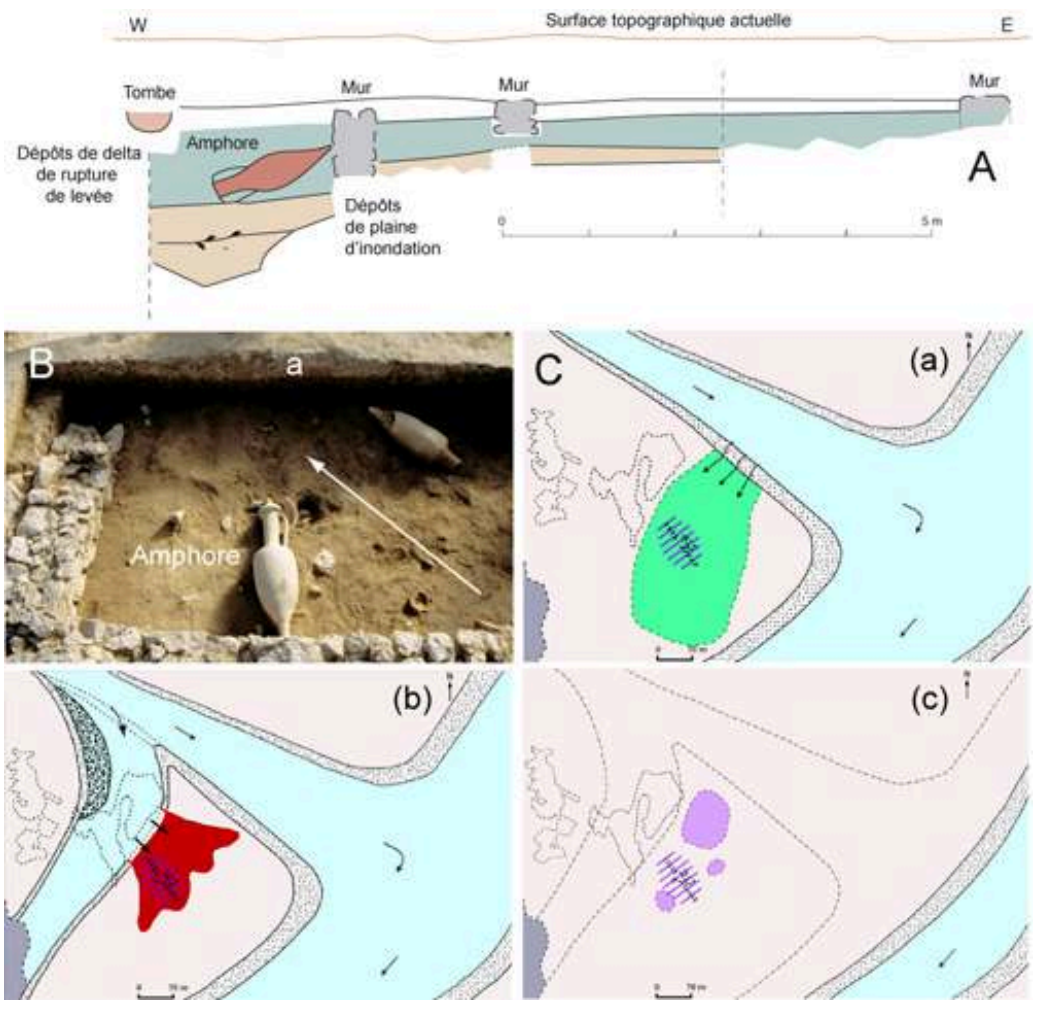

A : Détail de la coupe stratigraphique B montrant l'enfouissement des structures archéologiques (fin du ler siècle av. J.-C.) par les dépôts d'un delta de rupture de levée. B : Photographie de deux amphores (fin du ler siècle av. J.-C.) et de murs (fin du Ier siècle av. J.-C.) recouverts par (a) des dépôts alluviaux (plaine d'inondation, delta de rupture de levée ; cliché C. Landuré, 1999). La flèche indique la direction des paléoflux de crue. La présence d'une amphore fermée est le signe que le site a été rapidement abandonné, probablement après une grosse crue survenue à la fin du ler siècle av. J.-C. C : Cartes montrant l'évolution hydrographique autour du site archéologique. $A=V^{e}-$ er siècles av. J.-C. ; $b=$ fin du ler siècle av. J.-C. ; c = Ve siècle apr. J.-C. Noter la présence d'un delta de rupture de levée dont les dépôts limono-sableux recouvrent complètement le site habité à la fin du ler siècle av. J.-C. Cet événement catastrophique entraînera l'abandon de l'habitat jusqu'à 30 apr. J.-C. 
Figure 6. A : Interprétation paléohydrographique autour d'Aquilée durant l'Antiquité [d'après ArnaudFassetta et al., 2003]

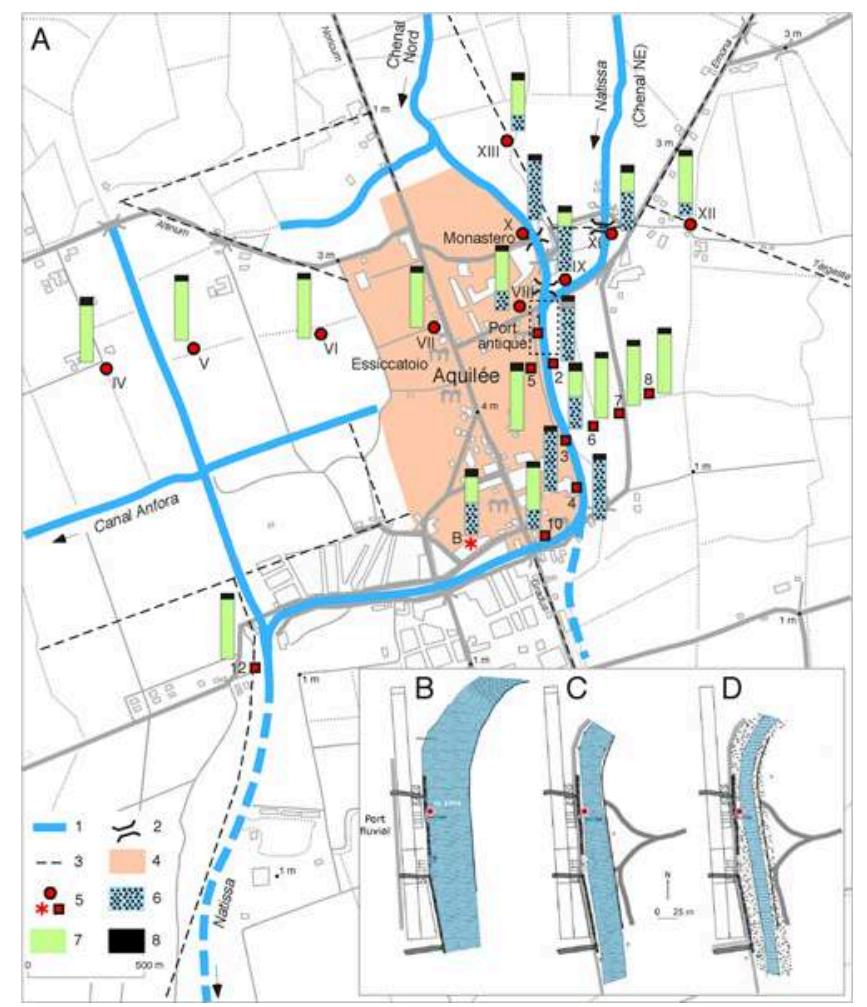

1 = paléochenal fluviatile romain ; 2 = pont romain ; $3=$ route romaine $; 4=$ cité romaine $; 5=$ sondages profonds ; 6 = dépôt de chenal $; 7$ = dépôt de plaine d'inondation; 8 = dépôt remanié. Le cadre localise les figures B, C et D. B, C et D : Evolution de la largeur du paléochenal du port antique d'Aquilée entre la fin du ler siècle apr. J.-C. et le haut Moyen Age [d'après Siché, thèse en cours]. A = Fin du ler siècle ; $B=$ IVe siècle ; $\mathrm{C}=$ haut Moyen Age.

$\mathrm{Au}$ total, les liens de causalité entre l'aléa et la vulnérabilité sociétale relèvent de processus multi-factoriels complexes qui n'ont pas toujours été strictement linéaires.

Dans le delta du Rhône, la pérennité des habitats ruraux dépend pour beaucoup de la manière dont s'exprime l'aléa dans la zone inondable (delta de rupture de levée, changement de tracé, remontée de nappe, hydraulicité et morphologie des chenaux). Si l'impact des changements de régime hydrologique et d'état du chenal fluviatile, comme entre le $\mathrm{I}^{\text {er }}$ siècle av. J.-C. et le $\mathrm{II}^{\mathrm{e}}$ siècle apr. J.-C., a eu des répercussions fortes sur l'habitat (abandon brutal, hiatus dans la fréquentation des sites) en raison d'un aléa trop pesant (« régime dominé par les crues »), cela n'a pas été une entrave à l'extension de l'occupation du sol dans le delta, qui s'est traduite au cours de la même période par la multiplication des zones habitées (i.e., plus grand nombre de sites créés) et ce, dans un contexte de progradation importante (i.e., gain de surface maximal) de l'aire deltaïque. De même à d'autres périodes, l'occupation a pu perdurer malgré la récurrence des inondations, ou à d'autres moments se rétracter sans que le risque d'inondation ne soit le véritable moteur de ce repli.

A Aquilée, on a pu montrer que la société romaine était finalement capable de maitriser et d'aménager le lit d'un fleuve quel que soit son état hydrologique (actif, moribond). Cependant, l'absence apparente de « déterminisme strict » du milieu doit être nuancée. En effet, le changement de tracé des principaux cours d'eau qui drainaient la ville, dans le courant de l'Antiquité, a eu sans aucun doute une incidence sur la fonction de grand 
port fluvial du site d'Aquilée, même si cette «fonction de site » est aussi dépendante des faits « historico-politiques».

Conclusions et perspectives en termes de dialectique sociétés-climat-dynamique fluviale-risque

La question des relations que les sociétés ont entretenues avec les cours d'eau et les plaines fluvio-deltaïques en général est complexe. Quelquefois, on sait qu'un certain déterminisme du milieu a pu prévaloir, comme Magny (1995) l'a montré autour des lacs jurassiens au cours de la seconde partie de l'Holocène. En Camargue, certaines phases de "régime dominé par les crues » ont également conduit à l'abandon brutal (mais temporaire ; résilience forte) de sites à cause d'inondations catastrophiques (exemple : La Capelière à la fin du Ir siècle av. J.-C.). Mais dans la plupart des cas, les recherches n'ont pas mis en évidence de déterminisme strict du milieu physique et du fonctionnement hydrosédimentaire des hydrosystèmes en particulier sur le comportement des sociétés. Dans le delta du Rhône, c'est au plus fort du risque fluvial que les bras actifs du Rhône (Saint-Ferréol, Ulmet) ont attiré et fixé les sociétés ; c'est au plus bas de leur activité que les sites habités se sont déplacés. A Aquilée, la société romaine aménage et développe le port fluvial quelles que soient les conditions hydrosédimentaires des cours d'eau. Le site est fondé à l'acmé du fonctionnement hydrodynamique. Le développement de la cité se poursuit malgré l'hydraulicité soutenue des paléochenaux et des crues récurrentes. C'est dans ce contexte que le port fluvial est devenu l'un des plus grands ports fluviaux méditerranéens de l'Antiquité.

\section{BIBLIOGRAPHIE}

Allinne C., 2005. Les villes antiques du Rhône et le risque fluvial. Gestion des inondations dans les villes romaines. L'exemple de la basse vallée du Rhône. Thèse, université de Provence (AixMarseille 1), 3 vol., 348 p., 141 p. et 198 p.

Arnaud-Fassetta G., 2000. Quatre mille ans d'histoire hydrologique dans le delta du Rhône. De l'âge du bronze au siècle du nucléaire. Grafigéo, 11, Collection mémoires et documents de l'UMR PRODIG, Paris, 229 p.

Arnaud-Fassetta G., 2006. Alloformations alluviales, morphodynamique fluviale et paléohydrologie, in Allée P., Lespez L. (Ed.) L'érosion entre société, climat et paléoenvironnement. Table ronde en l'honneur du Professeur René Neboit-Guilhot. Presses Universitaires Blaise Pascal, Collection Nature \& Sociétés, 3, Clermont-Ferrand, 35-46.

Arnaud-Fassetta G., 2007. L'hydrogéomorphologie fluviale, des hauts bassins montagnards aux plaines côtières : entre géographie des risques, géoarchéologie et géosciences. Dossier d'habilitation à diriger des recherches, université Paris-Diderot (Paris 7), volume $2:$ synthèse scientifique, $435 \mathrm{p}$.

Arnaud-Fassetta G., Landuré C., 2003. Hydroclimatic hazards, vulnerability of societies and fluvial risk in the Rhone Delta (Mediterranean France) from the Greek period to the Early Middle Ages, in Fouache E. (Ed.) The Mediterranean World Environment and History. Elsevier, Paris, 51-76. 
Arnaud-Fassetta G., Carre M.-B., Marocco R., Maselli Scotti F., Pugliese N., Zaccaria C., Bandelli A., Bresson V., Manzoni G., M.E. Montenegro, Morhange C., Pipan M., Prizzon A., Siché I., 2003. The site of Aquileia (northeastern Italy): example of fluvial geoarchaeology in a Mediterranean coastal plain, in Arnaud-Fassetta G., Provansal M. (Ed.) Deltas 2003. Géomorphologie : relief, processus, environnement, 4, 223-241.

Bravard J.-P., 2004. Le risque d'inondation dans le bassin du Haut Rhône : quelques concepts revisités dans une perspective géohistorique, in Burnouf J., Leveau P. (Ed.) Fleuves et marais, une histoire au croisement de la nature et de la culture. Sociétés préindustrielles et milieux fluviaux, lacustres et palustres : pratiques sociales et hydrosystèmes. CTHS, Paris, 397-408.

Bravard J.-P., Prestreau D. (Ed.), 1997. Dynamique du paysage-entretiens de géoarchéologie. Documents d'Archéologie en Rhône-Alpes, Lyon, 282 p.

Bravard J.-P., Salvador P.-G., 1999. Géomorphologie et sédimentologie des plaines alluviales, in Bravard J.-P., Cammas C., Nehlig P., Poupet P., Salvador P.-G., Wattez J. (Ed.) La géologie. Les sciences de la Terre. Editions Errance, Paris, 57-92.

Brown A.G., 1997. Alluvial geoarchaeology. Floodplain archaeology and environmental change. Cambridge University Press, Cambridge, 377 p.

Burnouf J., Leveau P. (Ed.), 2004. Fleuves et marais, une histoire au croisement de la nature et de la culture. Sociétés préindistrielles et milieux fluviaux, lacustres et palustres : pratiques sociales et hydrosystèmes. Comité des Travaux Historiques et Scientifiques, Paris, 493 p.

Butzer K.W., 1982. Archaeology as human ecology. Cambridge University Press, Cambridge.

Carcaud N., 2004. D'espace et de temps: un itinéraire de recherche et d'enseignement sur les anthroposystèmes fluviaux. Dossier d'habilitation à diriger des recherches, université d'Angers.

Chow V.T., 1959. Open channel hydraulics. McGraw-Hill, New York, 680 p.

Davidson D.A., Shackley M.L., 1976. Geoarchaeology. Duckworth, London.

Erskine W.D., Warner R.F., 1988. Geomorphic effects of alternating flood- and droughtdominated regimes on NSW coastal rivers, in Warner R.F. (Ed.) Fluvial Geomorphology of Australia. Academic Press, Sydney, 223-244.

Fouache E., 2001. Approche géoarchéologique en domaines balkaniques et méditerranéens : géomorphologie, paléo-environnements, histoire. Dossier d'habilitation à diriger des recherches, vol. 3, université Sorbonne (Paris 4), 274 p.

Fouache E. (Ed.), 2003. The Mediterranean world environment and history. Elsevier, Paris, 485 p. Horton R.E., 1932. Drainage basins characteristics. Trans. Am. Geophys. Union, 13, 350-361.

Leopold L.B., Wolman M.G., Miller J.P., 1964. Fluvial processes in geomorphology. Freeman and Co., San Francisco, 522 p.

Limerinos J.T., 1970. Determination of the Manning coefficient from measured bed roughness in natural channels. U.S. Geological Survey Water Supply Paper, 1898-B, 47 p.

Magny M., 1995. Une histoire du climat. Des derniers mammouths au siècle de l'automobile. Editions Errance, Paris, 176 p.

Manning R., 1891. On the flow of water in open channels and pipes. Transactions, Institution of Civil Engineers of Ireland, 20, 161-207.

Miall A.D., 1996. The geology of fluvial deposits. Sedimentary facies, basin analysis, and petroleum geology. Springer-Verlag, Berlin, $582 \mathrm{p}$. 
Morhange C., Provansal M. (Ed.), 2007. Géoarchéologie. Géomorphologie : relief, processus, environnement, $104 \mathrm{p}$.

Nanson G.C., Croke J.C., 1992. A genetic classification of floodplains. Geomorphology, 4, 459-486.

Needham S., Macklin M.G. (Ed.), 1992. Alluvial archaeology in Britain. Oxbow Monograph 27, Oxford, $277 \mathrm{p}$.

Passega R., 1957. Texture as characteristic of clastic deposition. American Association of Petroleum Geologists Bulletin, 41, 1952-1964.

Raban A., Holum K.G., 1996. Caesarea Maritima, a retrospective after two millenia. Brill LeidenNew York.

Rapp G., Gifford J.A., 1982. Archaeological geology. American scientist, 70, 45-53.

Roberts N., 1998. The Holocene. An environmental history. Blackwell, Oxford, 316 p.

Rotnicki A., 1991. Retroduction and palaeodischarges of meandering and sinuous alluvial rivers and its palaeohydroclimatic implications, in Starkel, L., Gregory, K.J., Thorne, J.B. (Ed.) Temperate Palaeohydrology. John Wiley and Sons, Chichester, 431-471.

Salvador P.-G., 2005. Géomorphologie et géoarchéologie des plaines alluviales (piémont alpin et nord de la France). Dossier d'habilitation à diriger des recherches, texte de synthèse, université des Sciences et Technologies de Lille 1, 298 p.

Siché I., en cours. Aléas fluviatiles et réponses des sociétés antiques dans les deltas de Méditerranée Nord-Occidentale. L'exemple de la plaine côtière d'Aquilée (Italie septentrionale). Thèse de géographie, cotutelle univ. Paris 7 et Trieste.

Siché I., Forte E., Prizzon S., Arnaud-Fassetta G., Fort M., 2006. Cartographie hydrogéomorphologique et paléochenaux fluviatiles en milieux profondément modifiés par les sociétés. L'exemple du port fluvial antique d'Aquilée dans la plaine du Frioul (Italie septentrionale, Adriatique). Actes du Colloque « Spatialisation et cartographie en hydrologie », Metz, 2004. Mosella, 3-4.

Strickler A. 1923. Beiträge zur Frage der Geschwindigkeitsformel und der Rauhigkeitszahl für Ströme, Kanäle und geschlossene Leitungen. Mitteilungen des Eidg. Amtes für Wasserwirtschaft, Bern, Switzerland, 16 p.

Vermeulen F., De Dapper M. (Ed.), 2000. Geoarchaeology of the landscapes of classical antiquity. Leiden, Stichting, Babesch, 233 p.

Vita-Finzi C., 1969. The Mediterranean valleys: geological changes in historical times. Cambridge, Cambridge University Press.

Williams G.P., 1978. Bankfull discharge of rivers. Water Resources Research, 14, 1141-1158.

\section{RÉSUMÉS}

Les recherches des hydrogéomorphologues ont des applications nombreuses dans le vaste champ des sciences géoarchéologiques. Elles fournissent des réponses précises sur la façon dont l'environnement des anciens lieux de passage et de vie humaine a évolué. Le propos n'est pas seulement de définir les causes des grands changements environnementaux, mais aussi de juger de la vulnérabilité sociétale face aux contraintes hydroclimatiques. Pour cela, les méthodes d'étude doivent nécessairement prendre en compte les trois facettes de la géomorphologie fluviale : la paléohydrographie, la paléohydrologie et la paléohydraulique. La pertinence de cette 
approche est montrée en milieu rural et urbain dans les plaines deltaïques du Rhône (France du Sud) et de l'Isonzo (Italie du Nord).

Current research led by hydrogeomorphologists has numerous applications in the vast field of geoarchaeological sciences. It brings precise answers on environmental characteristics around the ancient places of passage and human life. The goal is not only to define the causes of global environmental changes, but also to precise the links between river dynamics and human societies in terms of fluvial risk. Therefore, the studied methods should simultaneously take into account the three facets of the fluvial geomorphology, i.e., the palaeohydrography, the palaeohydrology, and the palaeohydraulics. The pertinence of this combinatorial approach is deduced from the work of the author led both in rural and urban areas of the deltaic plains of the Rhône (South of France) and Isonzo (northern Italy) rivers.

\section{INDEX}

Mots-clés : géoarchéologie fluviale, hydrogéomorphologie, aléa hydroclimatique, vulnérabilité sociétale, risque hydrologique

Keywords : fluvial geoarchaeology, hydrogeomorphology, hydroclimatic hazard, societal vulnerability, hydrological risk

\section{AUTEUR}

\section{GILLES ARNAUD-FASSETTA}

Gilles Arnaud-Fassetta, fassetta@univ-paris-diderot.fr, est Maître de conférences (HDR) en géographie physique à l'université Paris-Diderot (Paris 7). Il est membre du Conseil scientifique de l'UMR 8586 CNRS-PRODIG, Rédacteur adjoint de la revue Géomorphologie : relief, processus, environnement, vice-président du Groupe Français de Géomorphologie et membre du Bureau de la $23^{\mathrm{e}}$ section du CNU. Il a publié récemment en géoarchéologie fluviale :

- Arnaud-Fassetta G., Landuré C., 2003. Hydroclimatic hazards, vulnerability of societies and fluvial risk in the Rhone Delta (Mediterranean France) from the Greek period to the Early Middle Ages, in Fouache E. (Ed.), The Mediterranean World Environment and History, Elsevier, Paris, p. 51-76.

- Arnaud-Fassetta G. et al., 2003. The site of Aquileia (northeastern Italy): example of fluvial geoarchaeology in a Mediterranean coastal plain, in Arnaud-Fassetta G., Provansal M. (Ed.), Deltas 2003, Géomorphologie : relief, processus, environnement, 4, p. 223-241. 